\title{
REVIEW
}

\section{Discovery and development of the Polo-like kinase inhibitor volasertib in cancer therapy}

\author{
BT Gjertsen ${ }^{1,2,4}$ and P Schöffski ${ }^{3,4}$
}

\begin{abstract}
Owing to their integral involvement in cell cycle regulation, the Polo-like kinase (PIk) family, particularly PIk1, has emerged as an attractive therapeutic target in oncology. In recent years, several PIk1 inhibitors have been developed, with some agents showing encouraging results in early-phase clinical trials. This review focuses on volasertib (BI 6727; an investigational agent), a potent and selective Plk inhibitor. Volasertib has shown promising activity in various cancer cell lines and xenograft models of human cancer. Trials performed to date suggest that volasertib has clinical efficacy in a range of malignancies, with the most promising results seen in patients with acute myeloid leukemia (AML). Encouragingly, recent phase II data have demonstrated that volasertib combined with low-dose cytarabine (LDAC) was associated with higher response rates and improved event-free survival than LDAC alone in patients with previously untreated AML. Based on these observations, and its presumably manageable safety profile, volasertib is currently in phase III development as a potential treatment for patients with AML who are ineligible for intensive remission induction therapy. Given that many patients with AML are of an older age and frail, this constitutes an area of major unmet need. In this review, we discuss the biologic rationale for Plk1 inhibitors in cancer, the clinical development of volasertib to date in solid tumors and AML, and the future identification of biomarkers that might predict response to volasertib and help determine the role of this agent in the clinic.
\end{abstract}

Leukemia (2015) 29, 11-19; doi:10.1038/leu.2014.222

\section{INTRODUCTION}

The Polo-like kinases (Plks) comprise a family of five serine/ threonine protein kinases that have key roles in many processes involved in control of the cell cycle, including entry into mitosis, DNA replication and the stress response to DNA damage. However, Plk1 is deemed especially important and has been the focus of the majority of Plk research. Plk1, which is activated by another kinase, Aurora A, has multiple regulatory roles in the cell cycle, including the control of cell cycle progression into mitosis (Figure 1). ${ }^{1,2}$ Although the majority of studies highlight the role of Plk1 in mitosis, non-mitotic roles for Plk1 have also been suggested, including protection against apoptosis, ${ }^{3,4}$ and as a regulator of cancer cell invasiveness. ${ }^{5}$ Overexpression of Plk1 has been observed in a variety of solid tumors as well as in acute myeloid leukemia (AML), ${ }^{6-8}$ and has often been correlated with poor prognosis, disease stage, histologic grade, metastatic potential and survival., ${ }^{9,10}$ These observations have prompted research into the potential therapeutic application of Plk inhibitors in cancer.

One of the first Plk inhibitors to be developed was BI 2536. This selective, small-molecule, dihydropteridinone-derived, adenosine triphosphate-competitive kinase inhibitor of Plks 1-3 showed promise in preclinical experiments, validating Plks as potential drug targets in oncology.,11 Unfortunately, phase II trials in patients with relapsed/refractory solid tumors, ${ }^{12}$ non-small-cell lung cancer (NSCLC), ${ }^{13}$ small-cell lung cancer, ${ }^{14}$ chemotherapy-naive pancreatic cancer ${ }^{15}$ and relapsed/refractory $\mathrm{AML}^{16}$ demonstrated that BI 2536 had modest, if any, clinical activity and was associated with a generally manageable safety profile. These disappointing results were thought to be attributable to the pharmacokinetic (PK) profile of $\mathrm{BI} 2536$, particularly its relatively short terminal half-life $(\sim 50 \mathrm{~h} \text { in patients with advanced solid tumors })^{17}$ and low intratumoral exposure (as observed in hepatocellular carcinoma samples derived from murine xenograft models), despite a large distribution volume. ${ }^{18}$ Clinical development of BI 2536 was halted and continued development of novel Plk1 inhibitors focused on improving PK profiles to facilitate sustained exposure to tumor tissues.

In this review, we focus on the discovery and development of volasertib, a selective and potent cell cycle kinase inhibitor that induces Polo arrest and apoptosis by targeting PIk1. Volasertib is currently the most clinically advanced of the investigational Plk inhibitors and this review discusses its development to date in solid tumors and AML. In addition, we look forward to the possible development of biomarkers of volasertib response that could help drive future treatment decisions in individual patients.

\section{PRECLINICAL DEVELOPMENT OF VOLASERTIB}

Similar to BI 2536, a compound originally identified by screening a library of organic compounds for the capacity to inhibit the catalytic activity of Plk1, ${ }^{11}$ volasertib is a dihydropteridinone

\footnotetext{
${ }^{1}$ Centre for Cancer Biomarkers (CCBIO), Department of Clinical Science, University of Bergen, Bergen, Norway; ${ }^{2}$ Department of Internal Medicine, Haematology Section, Haukeland University Hospital, Bergen, Norway and ${ }^{3}$ Department of General Medical Oncology, Leuven Cancer Institute, University Hospitals Leuven, Katholieke Universiteit Leuven, Leuven, Belgium. Correspondence: Professor P Schöffski, Department of General Medical Oncology, Leuven Cancer Institute, University Hospitals Leuven, Katholieke Universiteit Leuven, Leuven, Belgium.

E-mail: patrick.schoffski@uzleuven.be

${ }^{4}$ These authors contributed equally to this work.

Received 19 February 2014; revised 5 June 2014; accepted 2 July 2014; accepted article preview online 16 July 2014 ; advance online publication, 15 August 2014
} 


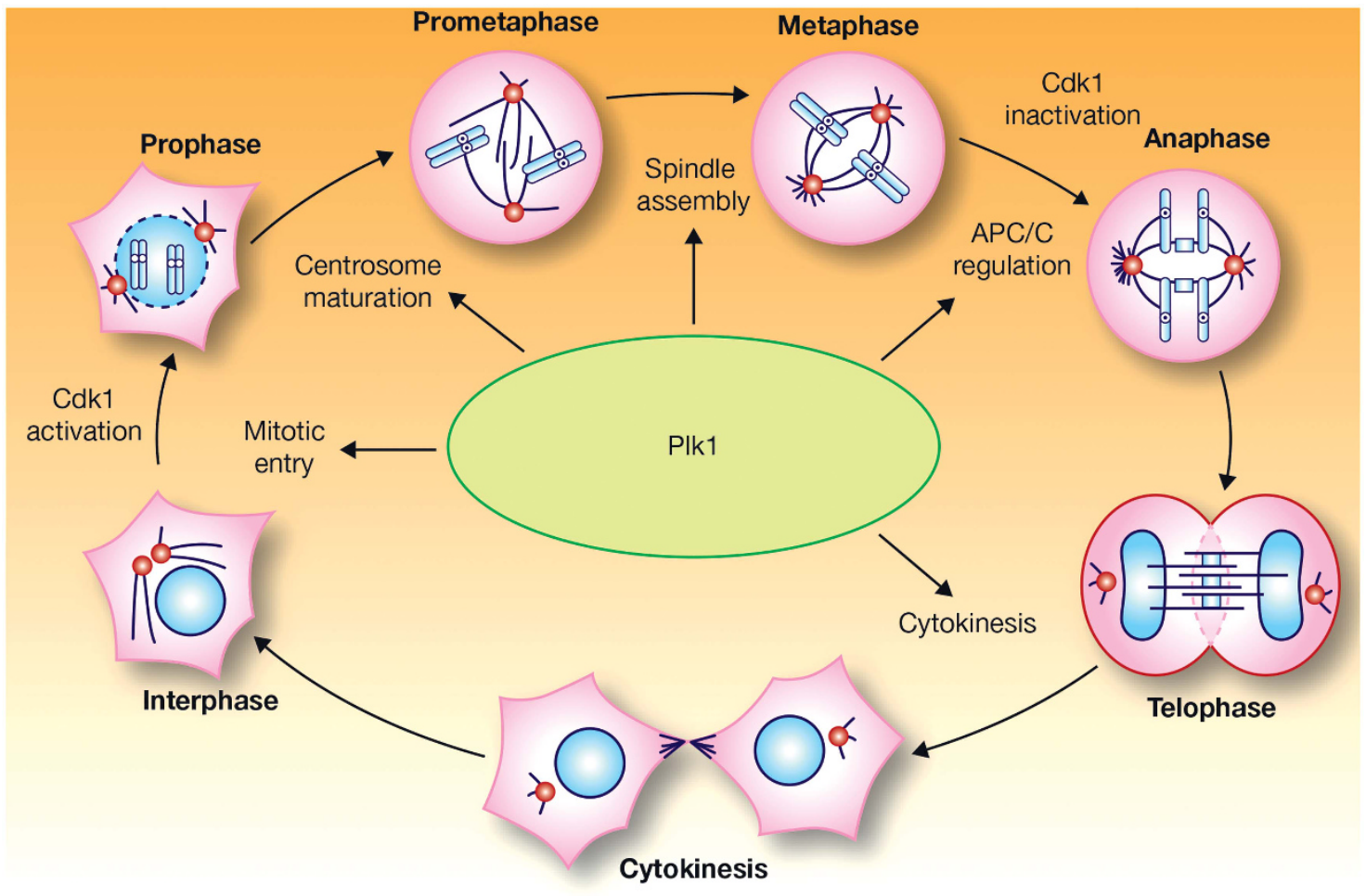

Figure 1. Functions of Plk1 during mitosis. APC/C, anaphase-promoting complex/cyclosome; Cdk1, cyclin-dependent kinase 1. Reprinted by permission from Macmillan Publishers Ltd: Nature Reviews Molecular Cell Biology (Barr et al. ${ }^{1}$ ), copyright 2004.

derivative and acts as a small-molecule, adenosine triphosphatecompetitive kinase inhibitor of Plk1. ${ }^{19}$ Both agents are potent and selective inhibitors of Plk1; however, volasertib was derived by tailoring the dihydropteridinone structure of BI 2536 based on the criteria of potency, selectivity, ability to inhibit cell proliferation in vitro, efficacy in xenograft models of cancer, drug metabolism and PK characteristics. ${ }^{19}$ The chemical structure and binding mode of volasertib to the adenosine triphosphate-binding pocket of human Plk1 are shown in Figures $2 a$ and $b$, respectively. It is important to note that volasertib is a unique chemical entity whose chemical structure and PK profile differ significantly compared with $\mathrm{BI} 2536$. As a result, any insights regarding efficacy and safety previously determined with BI 2536 cannot be simply transferred to volasertib.

Volasertib potently inhibits Plk1 (half-maximal inhibitory concentration $\left.\left(\mathrm{IC}_{50}\right) 0.87 \mathrm{nmol} / \mathrm{l}\right)$ as well as the two closely related kinases, Plk2 (IC $505 \mathrm{nmol} / \mathrm{I})$ and Plk3 (IC $505 \mathrm{nmol} / \mathrm{I})$, compared with $I_{50}$ values for $\mathrm{BI} 2536$ of $0.83,3.5$ and $9.0 \mathrm{nmol} / \mathrm{l}$, respectively, ${ }^{11}$ but shows no inhibitory activity against a panel of other unrelated kinases at concentrations up to $10 \mu \mathrm{mol} / \mathrm{l}$ (Table 1). ${ }^{19}$ Preclinical studies of volasertib in various tumor cell lines (colon, lung, melanoma, hematopoietic malignancies, prostate, urothelial and multiple pediatric tumors) have demonstrated inhibition of cell division that ultimately results in cell death. ${ }^{19-25}$ Targeting Plk with volasertib leads to a disruption of the mitotic spindle assembly resulting in a distinct mitotic arrest phenotype ('Polo arrest') in prometaphase, accumulation of phospho-histone H3 and formation of aberrant monopolar mitotic spindles followed by apoptosis (Figure 3). ${ }^{1,20,22,24,26}$ Volasertib has also been shown to inhibit the activity of the bromodomain and extraterminal family protein BRD4 in biochemical assays in vitro. ${ }^{27}$ BRD4, as well as fms-like tyrosine kinase 3 (FLT3), have been suggested as drivers in the pathogenesis of $A M L,{ }^{28,29}$ and preclinical studies of BRD4 inhibitors suggest the potential of these agents as antileukemic drugs. ${ }^{30,31}$ Although volasertib shows effects on BRD4, ${ }^{27}$ the cellular effects on BRD4 biomarker modulation are only observed at a concentration of $300 \mathrm{nM}$ suggesting that the effect on BRD4 may not be a relevant mechanism of action of volasertib.

Proteomic analyses of Plk-phosphorylated proteins using $\mathrm{BI}$ 2536 have identified hundreds of proteins normally affected by phosphorylation by Plk1, illustrating the importance of Plk1 in mitosis and other processes. ${ }^{32}$ Cellular proliferation is potently inhibited by volasertib, which acts on all proliferating cells, in a multitude of cancer cell lines, including colon (HCT116), lung (NCIH460), melanoma (BRO), non-Hodgkin lymphoma (GRANTA-519, Raji) and AML (HL-60, THP-1) cells with half-maximal effective concentration values of $11-37 \mathrm{nmol} / \mathrm{I}^{19}$ No correlation of Plk1 mRNA expression with sensitivity was observed in a data set based on 240 cancer cell lines derived from a variety of cancer indications treated with volasertib in vitro (unpublished data; Boehringer Ingelheim, Ingelheim, Germany). Volasertib also inhibited the growth and survival of cell lines derived from patients with pediatric acute lymphoblastic leukemia. ${ }^{25}$

In colon (HCT116) and lung (NCl-H460) xenograft tumor models, volasertib monotherapy was associated with reduced tumor growth, including growth delays and tumor regressions. ${ }^{19}$ Consistent with the in vitro data, volasertib treatment led to cell cycle arrest and apoptosis in tumor samples derived from tumorbearing mice. ${ }^{19}$ Volasertib concentrations measured in extracts from the tumors, multiple organs (brain, kidney, liver, lung and muscle) and plasma samples from these mice suggest good tissue penetration in all organs tested, although the central nervous system exposure is notably lower than the exposure observed for the other organs and does not exceed levels observed in the plasma. ${ }^{19}$ Marked antitumor activity and good tolerability were also observed in xenograft models of AML (Figure 4), human melanoma ${ }^{33}$ and various pediatric cancers. ${ }^{23,24}$ An improvement in antitumor control was observed with volasertib plus wholebody irradiation in a xenograft model of squamous cell carcinoma, likely as a result of concomitant cell cycle inhibition and cytotoxic effects of this combination. ${ }^{34}$ Preclinical PK data showed a high 
a<smiles>CC[C@@H]1C(=O)N(C)c2cnc(Nc3ccc(C(=O)NC4CCC(N5CCN(CC6CC6)CC5)CC4)cc3OC)nc2N1C(C)C</smiles>

b

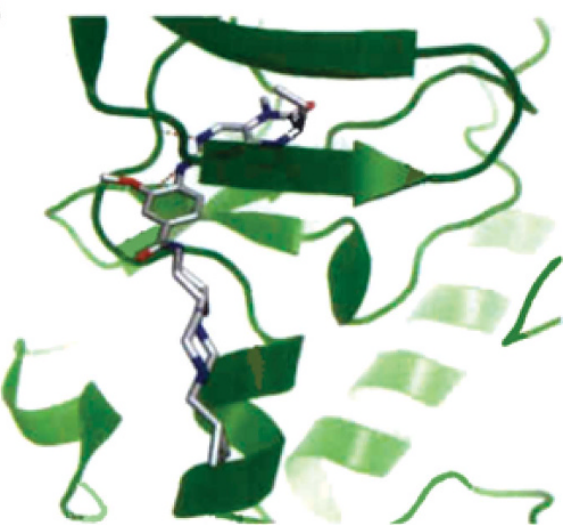

Figure 2. Chemical and X-ray structure of volasertib. Chemical structure (a) and binding mode (b) of volasertib in the adenosine triphosphatebinding pocket of human Plk1. Reprinted from Clinical Cancer Research, Copyright 2009, 15/9, 3094-3102, Rudolph et al. ${ }^{19}$ 'BI 6727, a Polo-like kinase inhibitor with improved pharmacokinetic profile and broad antitumor activity', with permission from AACR.

\begin{tabular}{|lc|}
\hline Table 1. Kinases not targeted by volasertib at concentrations up to \\
$10 \mu$ mol/l & \\
\hline Abl & MAPKAP-K2 \\
AMPK & MEK1 \\
Aurora A & Met \\
Aurora B & MKK1 \\
Axl & MSK1 \\
B-RAF & Nek6 \\
Btk & p38a \\
Cdk1/B1 & p38b \\
Cdk2/E & p38d \\
CHK1 & p38g \\
CK1 & PAK2 \\
CK2 & PDGFRa \\
C-RAF & PDGFRb \\
CSK & PDK1 \\
DYRK1A & PI3Ka \\
ECK & PKA \\
ErbB4 & PKBa \\
ERK2 & PKBb \\
FGFR1 & PKCa \\
FGFR3 & PRAK \\
FLT1 & Ret \\
FLT3 & ROCK2 \\
GSK3 & Ron \\
Hek & S6K \\
HER2 & SGK \\
HGFR & SrC \\
ITK & BIRK \\
JAK2 & Syk \\
JAK3 & Tie2 \\
JNK1 & VEGFR1 \\
Lck & VEGFR3 \\
Lyn & \\
\hline & \\
\hline
\end{tabular}

volume of distribution, indicating good tissue penetration, together with a long terminal half-life for volasertib compared with $\mathrm{BI} 2536 .^{19}$ Given these favorable PK properties that could potentially facilitate both intravenous (i.v.) and oral formulations, and promising preclinical efficacy and safety data, ${ }^{19}$ volasertib was prioritized for clinical development in both solid tumors and AML.

\section{CLINICAL DEVELOPMENT OF VOLASERTIB IN SOLID TUMORS}

Clinical trials of volasertib in solid tumors are listed in Table 2. The first-in-man trial was a phase I, dose-escalation study in 65 patients with progressive advanced or metastatic solid tumors, who received a single $1 \mathrm{~h}$ infusion of volasertib every 3 weeks. ${ }^{26}$ The most commonly reported drug-related adverse events (AEs) were anemia (22\% of patients), fatigue (15\%), neutropenia (15\%), thrombocytopenia (14\%), nausea (9\%), alopecia (9\%) and febrile neutropenia (8\%). Grade 3/4 AEs (24\%) were mostly related to hematologic toxicity (18\%), including dose-dependent neutropenia, both with and without infection, and thrombocytopenia. The main dose-limiting $\mathrm{AE}$ was neutropenia, and all cases of neutropenia and thrombocytopenia were reversible and manageable. The maximum-tolerated dose was established as $400 \mathrm{mg}$ administered intravenously on day 1 every 3 weeks; however, $300 \mathrm{mg}$ was selected for use in phase II trials owing to its generally manageable safety profile. PK analyses showed volasertib to have a large volume of distribution $(>4000 \mathrm{l})$, moderate clearance $(792 \mathrm{ml} / \mathrm{min})$ and a long half-life $(111 \mathrm{~h}){ }^{26}$ With no observed deviation from dose-linear PK behavior in the therapeutic relevant dose ranges, these data confirmed an improved PK profile of volasertib. ${ }^{19}$ The first-in-man trial also revealed signs of antitumor activity; three $(5 \%)$ patients (urothelial cancer, ovarian cancer and melanoma) achieved partial responses (PRs) and 26 (40\%) achieved stable disease, despite generally intense pretreatment (89\% had received $\geqslant 3$ prior chemotherapies). ${ }^{26}$

A comparable phase I study in patients with solid tumors has been undertaken in 52 Asian patients, with results largely consistent with those of the first-in-man trial. ${ }^{35}$ The maximumtolerated dose was identified as $300 \mathrm{mg}$ when given on day 1 every 3 weeks, and $150 \mathrm{mg}$ when administered on days 1 and 8 every 3 weeks. Dose-limiting toxicities were reversible thrombocytopenia, neutropenia and febrile neutropenia. Volasertib displayed some antitumor activity, with two (4\%) patients (ureteral cancer and melanoma) achieving PR, while PK results showed a half-life of $\sim 107 \mathrm{~h}$ and a large volume of distribution (4500 l). ${ }^{35}$ A separate phase I trial in Japanese patients with solid tumors is ongoing (Table 2).

A phase II trial of volasertib was undertaken in 50 patients with metastatic urothelial cancer following platinum failure. ${ }^{36}$ Although patients were heavily pretreated, volasertib demonstrated antitumor activity with seven (14\%) patients achieving PR and 13 (26\%) achieving stable disease as best response. The most common grade $3 / 4$ AEs were neutropenia (28\%), thrombocytopenia $(20 \%)$ and anemia $(16 \%)$; there was no evidence of cumulative toxicity. ${ }^{36}$

Two phase II trials of volasertib are ongoing in advanced ovarian cancer and advanced NSCLC (Table 2). Preliminary results from the ovarian cancer study indicate that single-agent volasertib shows antitumor activity comparable with that achieved with investigator's choice single-agent chemotherapy, while AEs were mainly hematologic and manageable. ${ }^{37}$ Initial data from the NSCLC study 

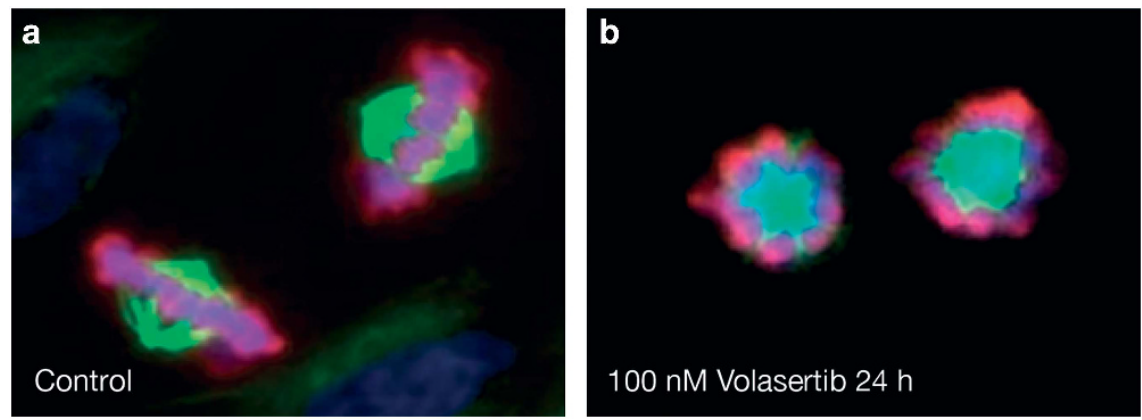

Figure 3. Volasertib prevents bipolar spindle formation, inducing cell cycle arrest in the early $\mathrm{M}$ phase. Immunofluorescence analysis of $\mathrm{NCl}-\mathrm{H} 460 \mathrm{NSCLC}$ cells treated for $24 \mathrm{~h}$ with either (a) $0.1 \%$ dimethyl sulfoxide or (b) $100 \mathrm{~nm} / \mathrm{l}$ volasertib. Cells were fixed and stained with 4', 6-diamidino-2-phenylindole (to stain DNA; blue), anti-tubulin (to stain spindles; green) and anti-phosphoSer10 histone H3 (pink). Volasertib treatment resulted in an accumulation of mitotic cells with monopolar spindles in which the kinetochores were not properly attached to the spindle-a cellular phenotype termed 'Polo arrest'. Reprinted from Clinical Cancer Research, Copyright 2009, 15/9, 3094-3102, Rudolph et al. ${ }^{19}$ 'BI 6727, a Polo-like kinase inhibitor with improved pharmacokinetic profile and broad antitumor activity', with permission from AACR.
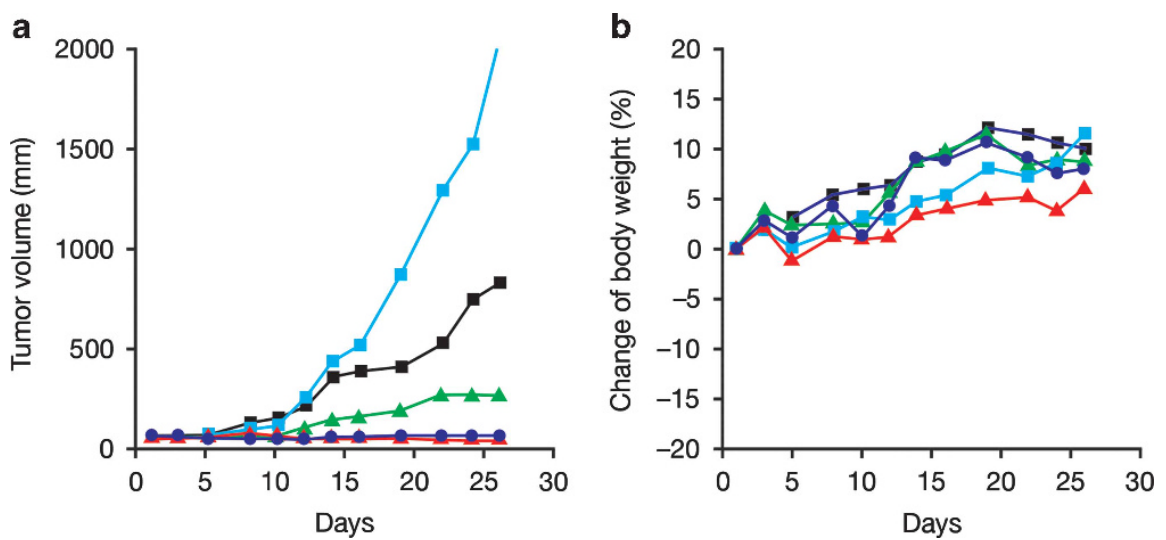

Figure 4. Efficacy and tolerability of volasertib in human AML xenograft model. Nude mice bearing established subcutaneous MV4-11 AML tumors with an average size of $\sim 65 \mathrm{~mm}^{3}$ were treated intravenously for 4 weeks with either vehicle (light blue squares) or volasertib at $40 \mathrm{mg} / \mathrm{kg}$ (blue circles), $20 \mathrm{mg} / \mathrm{kg}$ (green triangles), or $10 \mathrm{mg} / \mathrm{kg}$ once a week (black squares), or at $20 \mathrm{mg} / \mathrm{kg}$ two times a week on consecutive days (red triangles). Median tumor volumes of eight animals per treatment group (a) and median body weight change as \% of initial body weight (b) are shown. Efficacy has also been demonstrated in three disseminated AML models (MV4-11 (FLT3-ITD/FLT3-ITD), Molm-13 (FLT3-ITD/wild-type FLT3) and THP-1 (wild-type FLT3/wild-type FLT3) cell lines).

in patients with relapsed or refractory NSCLC after platinum-based first-line therapy show a lack of improvement in progression-free survival or overall response rate with combination volasertib and pemetrexed compared with single-agent pemetrexed. ${ }^{38}$

Although some patients with solid tumors have responded well to single-agent volasertib, with some patients able to achieve prolonged durations of response, overall antitumor activity has been modest in trials performed to date. ${ }^{26,36}$ Combination therapy is a potential option to improve efficacy, and volasertib has been combined with platinum chemotherapy to investigate this approach. In a phase I study in 61 heavily pretreated patients with advanced solid tumors, combination treatment was generally well tolerated, suggesting that volasertib is suitable for coadministration with cytotoxic chemotherapy at full single-agent doses. $^{39,40}$ Five (8\%) patients achieved PR, while 27 (44\%) achieved stable disease or PR. Additional studies in advanced solid tumors are investigating volasertib combined with other agents, including trials involving the kinase inhibitors afatinib (NCT01206816) and nintedanib (NCT01022853), in which antitumor activity has been observed. Responses were observed in $2 / 29$ patients treated with volasertib plus afatinib (PRs in both patients with NSCLC and head and neck cancer, respectively) and 2/30 patients treated with volasertib plus nintedanib (a complete response $(C R)$ in a patient with breast cancer and a PR in a patient with NSCLC). 41,42

\section{AN UNMET NEED FOR NEW THERAPIES IN AML}

Treatment guidelines are well established for $\mathrm{AML}^{43-45}$ a malignancy that can be challenging to manage in many cases. ${ }^{46}$ Induction chemotherapy with an anthracycline and cytarabine, as in the widely used ' $3+7$ ' regimen, is standard; however, many patients with AML are of an older age and unable to tolerate this intensive treatment. ${ }^{44,45}$ Data from the past three decades, in which anthracyclines plus cytarabine was the most commonly used therapy for AML, demonstrate that little progress has been made in improving overall survival in patients aged $\geqslant 60$ years compared with those aged $<60$ years. ${ }^{47,48}$ In addition, there are various cytogenetic subtypes of $A M L$ that strongly influence response to treatment, and therapeutic resistance associated with adverse cytogenetics is common. ${ }^{44,45}$ Hypomethylating agents or low-dose cytarabine (LDAC) are options for some older, less-fit patients. Azacitidine has demonstrated a survival benefit vs conventional care regimens in older patients with $A M L$ and 20-30\% blasts; however, no survival benefit has yet been determined with azacitidine in patients with $\mathrm{AML}$ and $>30 \%$ blasts. $^{49}$ Similarly, a randomized trial of LDAC vs palliative treatment in patients not considered eligible for intensive therapy demonstrated a better $\mathrm{CR}$ rate and improved survival with LDAC, but no benefit was seen in patients with adverse cytogenetics. ${ }^{50}$ In contrast, decitabine demonstrated improved $\mathrm{CR}$ rates, but primary 


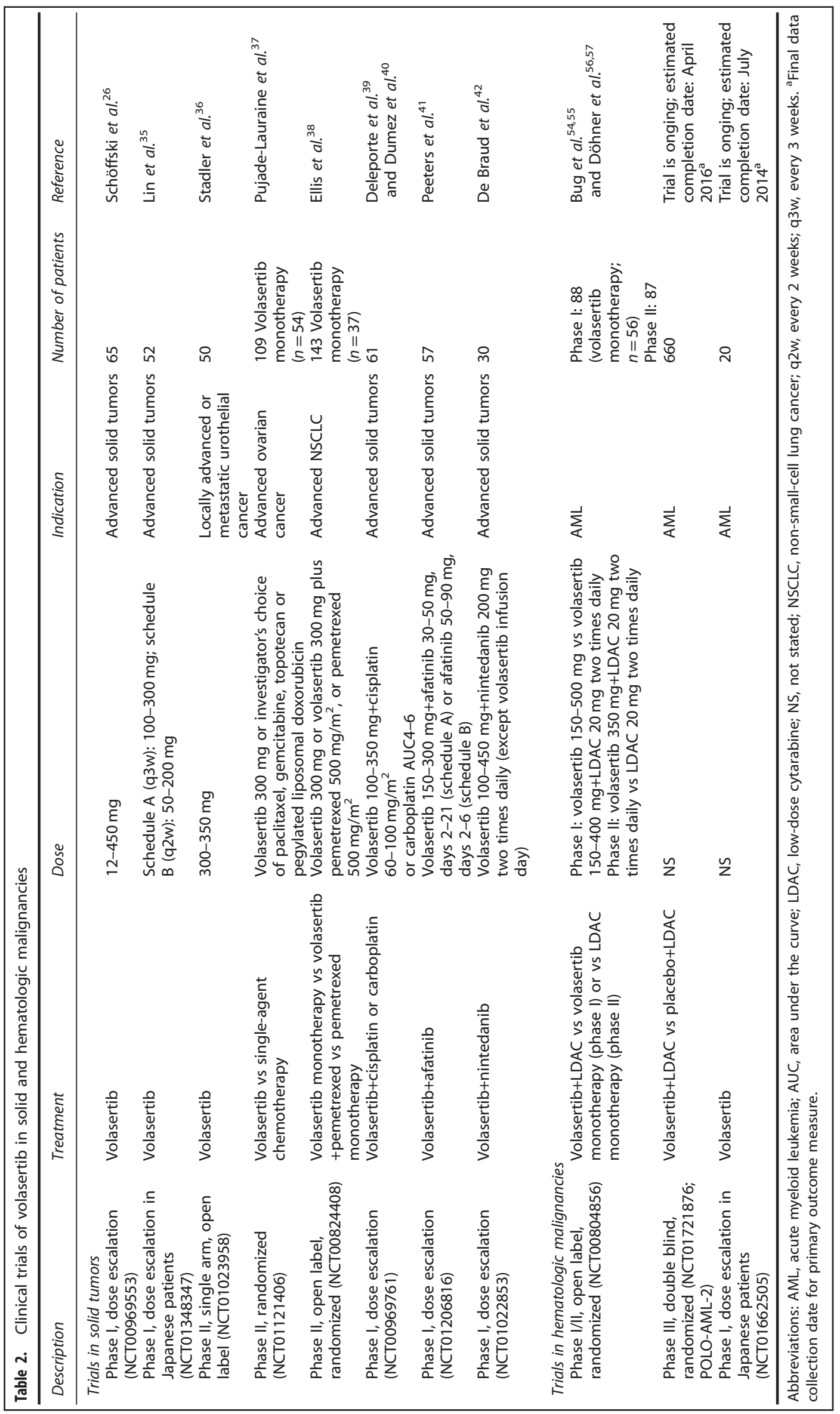


analysis did not show a significant survival benefit compared with either supportive care or LDAC in older patients with newly diagnosed AML. ${ }^{51}$ More efficacious treatments are urgently needed, ${ }^{46,52}$ and as cell cycle inhibitors may be particularly useful in rapidly proliferating malignancies such as $A M L$, there is a clear rationale for investigating Plk inhibitors in this setting. ${ }^{8}$ In this context, in vitro studies have shown that Plk1 is highly expressed in leukemic cell lines and tumor cell samples derived from patients with AML compared with normal hematopoietic progenitor cells. ${ }^{7,53}$ Furthermore, leukemic cells were shown to be more sensitive to Plk1 inhibition, as demonstrated by a marked decrease in cell proliferation, compared with normal progenitor cells. ${ }^{7}$

\section{CLINICAL DEVELOPMENT OF VOLASERTIB IN AML}

The clinical development of volasertib in AML is well underway; reporting, ongoing and planned clinical trials are listed in Table 2. A phase I/II study evaluated the safety, efficacy and PKs of volasertib plus LDAC and volasertib monotherapy in patients with $A M L$ ineligible for intensive remission induction therapy. ${ }^{54-57}$ This trial was performed in two parts: a phase I part and a phase lla part. In the phase I part of the trial, volasertib was investigated in combination with LDAC $(n=32)$ or as a monotherapy $(n=56)$ in patients with relapsed/refractory $A M L$ to determine the maximum-tolerated dose. In the phase II part, the combination of volasertib plus LDAC $(n=42)$ was compared with LDAC monotherapy $(n=45)$ in patients with previously untreated AML to explore the efficacy of the combination schedule in comparison with LDAC monotherapy. The phase I, dose-escalation part of the trial, conducted in patients with relapsed/refractory $A M L$, identified the recommended dose of volasertib as $450 \mathrm{mg}$ every second week as a single agent and $350 \mathrm{mg}$ every second week in combination with LDAC. ${ }^{54-56}$ At volasertib monotherapy doses $\geqslant 350 \mathrm{mg}$, antileukemic activity was observed, with 5/43 (12\%) patients achieving a complete remission with incomplete blood count recovery $(\mathrm{CRi}) .^{56}$ In the volasertib plus LDAC combination arm, 7/32 patients achieved a CR or CRi. ${ }^{55}$ In the phase II part of the trial, preliminary data demonstrated a substantially higher objective response rate with volasertib $(350 \mathrm{mg})$ plus LDAC vs LDAC alone with a CR/CRi rate of $31.0 \%$ vs $13.3 \% \quad(P=0.052)$. Furthermore, subgroup analyses indicate that responses in the volasertib plus LDAC treatment arm occurred across all genetic groups (including adverse genetics). A CR was observed in $1 / 5$ patients with FLT3-internal tandem duplication (ITD) and 3/7 patients with nucleophosmin 1 mutations treated with volasertib plus LDAC. ${ }^{57}$ Median event-free survival (5.6 vs 2.3 months; $P=0.021$ ) and overall survival (8.0 vs 5.2 months; $P=0.047$ ) was also significantly improved for volasertib plus LDAC vs LDAC alone. ${ }^{57}$ While toxicity was increased with combination therapy, presumably owing to the myelosuppressive effect of volasertib, there was no evidence of increased early mortality for the combination regimen vs LDAC alone. ${ }^{57}$ PK analyses showed that volasertib exhibited multicompartmental behavior with moderate clearance, a large volume of distribution and a long terminal halflife, ${ }^{55-57}$ as seen in patients with advanced solid tumors. ${ }^{26}$

These encouraging data from the phase II part of this AML trial has prompted the initiation of a phase III trial of volasertib in AML (POLO-AML-2). This trial is designed to evaluate, in a randomized, double-blind setting, the efficacy and safety of volasertib plus LDAC vs placebo plus LDAC in 660 patients aged $\geqslant 65$ years with previously untreated $A M L$ who are ineligible for intensive remission induction therapy (NCT01721876). The primary end point of POLO-AML-2 is an objective response (CR and CRi), and the study is expected to be completed in early 2016 (final data collection date for primary outcome measure). A volasertib monotherapy, dose-escalation, phase I trial in Japanese patients with AML (NCT01662505) and another phase I study of volasertib plus decitabine, a hypomethylating agent approved by the European Medicines Agency for the treatment of patients $\geqslant 65$ years with AML, are ongoing (NCT02003573). Volasertib is also being investigated in a phase I trial in combination with azacitidine in patients with myelodysplastic syndromes or chronic myelomonocytic leukemia who are ineligible for high-intensity therapy (NCT01957644).

\section{POTENTIAL BIOMARKERS OF VOLASERTIB SENSITIVITY}

As discussed above, treatment with volasertib as a monotherapy and in combination with other agents resulted in significant clinical benefits, including prolonged durations of response, for subgroups of patients with solid tumors. ${ }^{26,35-37,39-42}$ In AML, volasertib in combination with LDAC resulted in a CR/CRi rate of $31 \%$ of patients in a randomized, phase II trial of patients with previously untreated AML. ${ }^{57}$ As not all patients respond to volasertib therapy, predictive biomarkers are of interest to determine and/or predict drug activity and ultimately optimize therapy options for patients.

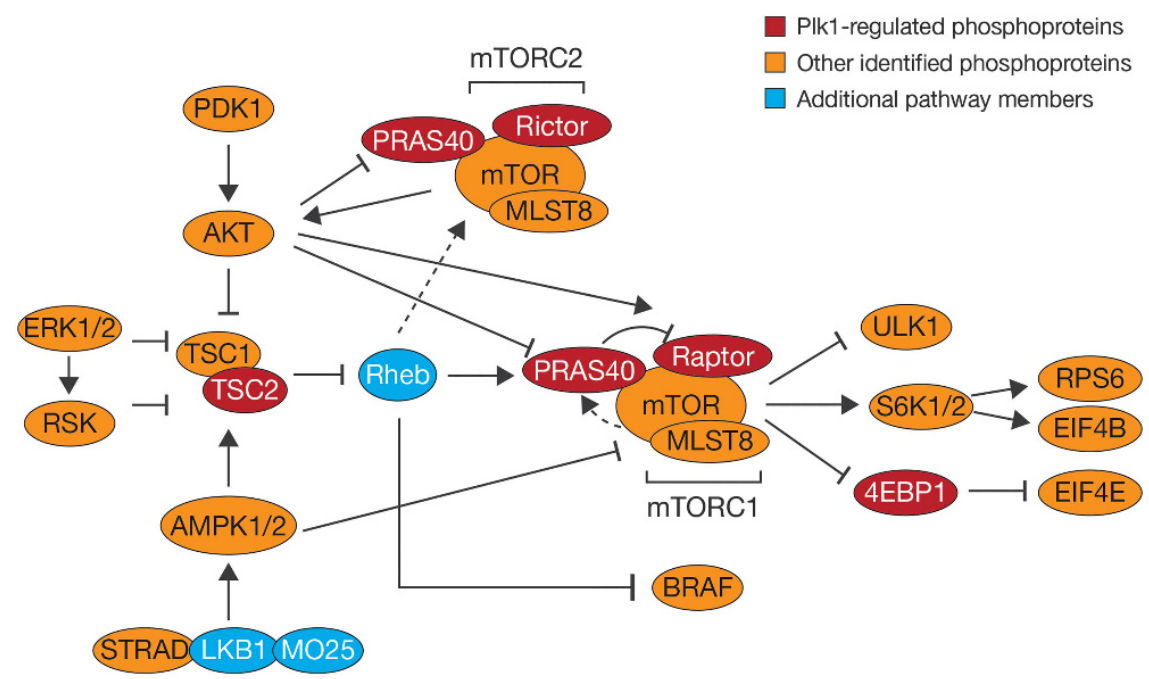

Figure 5. Example of non-mitotic functions of Plk1: effects on the mTOR pathway. Reproduced from Oppermann et al. ${ }^{63}$ with kind permission from the American Society for Biochemistry and Molecular Biology. 
Proteomic analyses have identified proteins normally phosphorylated by Plk1, including many that are not involved with mitosis. ${ }^{32}$ These, and other proteins such as heat-shock protein $70{ }^{58}$ may be worth investigating as potential biomarkers of volasertib sensitivity. There is also a possibility that Plk1-regulated phosphoproteins may be implicated in serine/threonine kinase activation of the mammalian target of rapamycin (mTOR) pathway and a molecular and functional link between Plk1 and the mTOR pathway has been shown in AML. ${ }^{59}$ As the mTOR pathway displays aberrant regulation in hematologic malignancies such as $A M L,{ }^{60-62}$ a further range of potential downstream markers could be investigated for sensitivity to volasertib (Figure 5). ${ }^{32,63}$ In this regard, it is noteworthy that a proteomics study in AML identified a signature of four phosphorylated proteins that act as predictors for response to the FLT3-targeted tyrosine kinase inhibitor, quizartinib. ${ }^{64}$ Proteomics of AML is still in its infancy, ${ }^{65}$ but may allow delineation of defined signal transduction and tumor suppressor pathways in single cells or cell extract assay: ${ }^{66,67}$ sample formats that may be relevant for future clinical trials of Plk1-targeting therapy. Relevant markers might be also identified based on comprehensive genomic, epigenomic and transcriptomic analyses.

Other possible biomarkers may include the breast cancer susceptibility protein, BRCA2, and the tumor suppressor protein, p53. Given that Plk1 has been shown to phosphorylate BRCA2, ${ }^{68}$ the presence or absence of this Plk1 substrate could potentially have some impact on volasertib susceptibility in solid tumors. In another study, $\mathrm{p} 53$ has been suggested as an in vitro predictive biomarker for the Plk1 inhibitor, GSK461364A, with the loss of p53 function resulting in sensitivity to the drug in a panel of human cancer cell lines. ${ }^{69}$ However, a separate study on colon cancer cell lines with volasertib showed no similar relationship. ${ }^{70}$ Clearly, the relationship between PIk1 and p53 is complex and further work is required to elucidate the full mechanism of their interaction in different malignancies.

Recently, volasertib has been reported to inhibit BRD4 binding at double-digit nanomolar concentrations in biochemical and binding assays; however, cellular effects on a BRD4 pharmacodynamic biomarker were only observed at concentrations of $300 \mathrm{~nm}^{27}$ In published works, inhibitors of BRD4 appear more effective in nucleophosmin 1 or DNA (cytosine-5)-methyltransferase 3-mutated AML cells, ${ }^{71,72}$ but a deeper understanding of the role of BRD4 as epigenetic modulator in AML is still missing. The concept of dual Plk1 and BRD4 inhibition may be interesting in light of ongoing trials with epigenetic modulators. ${ }^{73}$ The histone deacteylase inhibitor valproic acid has been shown to upregulate trimethylation of histone $\mathrm{H} 3$ lysine 27 in $\mathrm{AML}$ cells, ${ }^{74}$ and may be an interesting drug for volasertib combination therapy.

\section{CONCLUSIONS AND FUTURE PERSPECTIVE}

To date, the promising therapeutic effects of volasertib have been most clearly observed in patients with $A M L$ when given in combination with LDAC. In part, this may be due to the high mitotic index of the malignancy and its high expression of Plk1, combined with the balanced efficacy and safety profiles of this drug and its favorable PK characteristics. From a drug exposure perspective, the concentration of volasertib in the vicinity of malignant hematopoietic cells in the blood/bone marrow of AML patients may differ significantly from that in the tissue surrounding cancerous cells in solid tumors. Indeed, the malignant cells in $A M L$ reside in the same vascular system that delivers the drug into the body. Even small molecules like volasertib may have limited distribution in solid tumors owing to heterogeneous blood supply, elevated interstitial pressure and large transport distances in the interstitium, ${ }^{75}$ although this may be partially eliminated by the large distribution volume observed with volasertib. ${ }^{26}$ Finally, vigorous proliferation of malignant cells may be observed in
AML, making this disease more susceptible to Plk1 inhibitors than solid tumors, whose cells tend to display a slower growth rate.

In the 25 years since intensive induction regimens became standard therapy for $\mathrm{AML}$, little progress has been made in improving overall survival in patients aged $\geqslant 60$ years. ${ }^{47,48}$ An urgent need therefore exists for development of better therapies for $A M L$, especially in older patients. The use of volasertib in combination therapy is a promising option requiring further exploration in this setting, particularly in previously untreated patients with $A M L$ who are ineligible for intensive induction therapy. Results of the POLO-AML-2 phase III AML trial will provide useful insights into the potential utility of this approach in this patient population.

\section{CONFLICT OF INTEREST}

BG has received compensation as a member of the scientific advisory board of Clavis Pharma AS (2012), BerGenBio AS and Boehringer Ingelheim GmbH and owns shares in Kinn Therapeutics AS and Alden Cancer Therapy AS. PS declares no conflict of interest.

\section{ACKNOWLEDGEMENTS}

We thank Dr Dorothea Rudolph (Boehringer Ingelheim) for her kind and to the point contributions to this review. We are fully responsible for all content and editorial decisions, were involved at all stages of manuscript development and have approved the final version of this review, which reflects the authors' interpretation and conclusions. Medical writing assistance, supported financially by Boehringer Ingelheim, was provided by Victoria Robb of GeoMed, part of KnowledgePoint360, an Ashfield company, during the preparation of this review. Boehringer Ingelheim was given the opportunity to check the data for factual accuracy only.

\section{REFERENCES}

1 Barr FA, Sillje HH, Nigg EA. Polo-like kinases and the orchestration of cell division. Nat Rev Mol Cell Biol 2004; 5: 429-440.

2 Medema RH, Lin CC, Yang JC. Polo-like kinase 1 inhibitors and their potential role in anticancer therapy, with a focus on NSCLC. Clin Cancer Res 2011; 17: 6459-6466.

3 Liu X, Erikson RL. Polo-like kinase (Plk)1 depletion induces apoptosis in cancer cells. Proc Natl Acad Sci USA 2003; 100: 5789-5794.

4 Tamura Y, Simizu S, Muroi M, Takagi S, Kawatani M, Watanabe N et al. Polo-like kinase 1 phosphorylates and regulates $\mathrm{BCl}-\mathrm{x}(\mathrm{L})$ during pironetin-induced apoptosis. Oncogene 2009; 28: 107-116.

5 Rizki A, Mott JD, Bissell MJ. Polo-like kinase 1 is involved in invasion through extracellular matrix. Cancer Res 2007; 67: 11106-11110.

6 Holtrich U, Wolf G, Brauninger A, Karn T, Bohme B, Rubsamen-Waigmann $\mathrm{H}$ et al. Induction and down-regulation of PLK, a human serine/threonine kinase expressed in proliferating cells and tumors. Proc Natl Acad Sci USA 1994; 91: 1736-1740.

7 Renner AG, Dos SC, Recher C, Bailly C, Créancier L, Kruczynski A et al. Polo-like kinase 1 is overexpressed in acute myeloid leukemia and its inhibition preferentially targets the proliferation of leukemic cells. Blood 2009; 114: 659-662.

8 Tsykunova G, Reikvam H, Ahmed AB, Nepstad I, Gjertsen BT, Bruserud O. Targeting of polo-like kinases and their cross talk with Aurora kinases-possible therapeutic strategies in human acute myeloid leukemia? Expert Opin Investig Drugs 2012; 21: 587-603.

9 Weiss L, Efferth T. Polo-like kinase 1 as target for cancer therapy. Exp Hematol Oncol 2012; 1: 38

10 Strebhardt K. Multifaceted polo-like kinases: drug targets and antitargets for cancer therapy. Nat Rev Drug Discov 2010; 9: 643-660.

11 Steegmaier $M$, Hoffmann $M$, Baum $A$, Lénárt $P$, Petronczki $M$, Krssák $M$ et al. BI 2536, a potent and selective inhibitor of polo-like kinase 1, inhibits tumor growth in vivo. Curr Biol 2007; 17: 316-322.

12 Schöffski P, Blay JY, De GJ, Brain E, Machiels JP, Soria JC et al. Multicentric parallel phase II trial of the polo-like kinase 1 inhibitor $\mathrm{BI} 2536$ in patients with advanced head and neck cancer, breast cancer, ovarian cancer, soft tissue sarcoma and melanoma. The first protocol of the European Organization for Research and Treatment of Cancer (EORTC) Network Of Core Institutes (NOCl). Eur J Cancer 2010; 46: 2206-2215.

13 Sebastian M, Reck M, Waller CF, Kortsik C, Frickhofen N, Schuler M et al. The efficacy and safety of BI 2536, a novel Plk-1 inhibitor, in patients with stage IIIB/IV non-small cell lung cancer who had relapsed after, or failed, chemotherapy: results from an open-label, randomized phase II clinical trial. $J$ Thorac Oncol 2010; 5: 1060-1067. 
14 Chu Q, Gandhi L, Stephenson J, Johnson BE, Govindan R, Bradford D et al. An open-label phase II trial of the Plk1 inhibitor BI 2536, in patients with sensitive relapse small cell lung cancer. J Thorac Oncol 2009; 4(Part2): 200.

15 Mross K, Dittrich C, Aulitzky WE, Strumberg D, Schutte J, Schmid RM et al. A randomised phase II trial of the Polo-like kinase inhibitor BI 2536 in chemonaive patients with unresectable exocrine adenocarcinoma of the pancreas-a study within the Central European Society Anticancer Drug Research (CESAR) collaborative network. Br J Cancer 2012; 107: 280-286.

16 Müller-Tidow C, Bug G, Lübbert M, Krämer A, Krauter J, Valent $P$ et al. A randomized, open-label, phase $\mathrm{I} / \mathrm{II}$ trial to investigate the maximum tolerated dose of the Polo-like kinase inhibitor BI 2536 in elderly patients with refractory/relapsed acute myeloid leukaemia. Br J Haematol 2013; 163: 214-222.

17 Hofheinz RD, Al-Batran SE, Hochhaus A, Jäger E, Reichardt VL, Fritsch $\mathrm{H}$ et al. An open-label, phase I study of the polo-like kinase-1 inhibitor, BI 2536, in patients with advanced solid tumors. Clin Cancer Res 2010; 16: 4666-4674.

18 Haupenthal J, Bihrer V, Korkusuz H, Kollmar O, Schmithals C, Kriener S et al. Reduced efficacy of the Plk1 inhibitor BI 2536 on the progression of hepatocellular carcinoma due to low intratumoral drug levels. Neoplasia 2012; 14: 410-419.

19 Rudolph D, Steegmaier M, Hoffmann M, Grauert M, Baum A, Quant J et al. BI 6727, a Polo-like kinase inhibitor with improved pharmacokinetic profile and broad antitumor activity. Clin Cancer Res 2009; 15: 3094-3102.

20 Paller CJ, Wissing MD, Rosmus NS, Hammers H, Rudin CM, Carducci MA et al. Preclinical synergy of Plk1 inhibitors and HDACls. Cancer Res 2011; 71: 2557.

21 Wissing MD, Rosmus N, Gonzalez M, Hammers H, Carducci MA, Kachhap SK. Targeting prostate cancer through a combination of Polo-like kinase inhibitors and histone deacetylase inhibitors. Cancer Res 2010; 70: 5414.

22 Lin CC, Lin Y-M, Tsai Y-C, Pu Y-S, Cheng A-L, Yang JC. Combined cytotoxic effects of volasertib and cisplatin in urothelial carcinoma cells. Cancer Res 2012; 72: 3725

23 Smith M, Keir S, Maris J, Kolb A, Reynolds P, Kang M et al. Pediatric Preclinical Testing Program (PPTP) evaluation of volasertib (BI 6727), a Polo-like kinase (PLK) inhibitor. Cancer Res 2012; 72: LB-317.

24 Lanvers-Kaminsky C, Abbou S, Daudigeos-Dubus E, Molenaar J, Verschuur A, Caron $\mathrm{H}$ et al. Anti-tumor activity of the PLK inhibitor volasertib (BI 6727) and the aurora kinase inhibitor BI 811283 in pediatric malignancies. Eur J Cancer 2012; 48: 78 .

25 Jayanthan A, Cooper T, Dunn SE, Lewis VA, Narendran A. Cooperative lethality of Polo-like kinases (PLK) and Aurora kinases (AK) in refractory pediatric leukemia. Blood 2012; 120: 3573

26 Schöffski P, Awada A, Dumez H, Gil T, Bartholomeus S, Wolter P et al. A phase I, dose-escalation study of the novel Polo-like kinase inhibitor volasertib (BI 6727) in patients with advanced solid tumours. Eur J Cancer 2012; 48: 179-186.

27 Ciceri P, Müller S, O'Mahony A, Fedorov O, Filippakopoulos P, Hunt JP et al. Dual kinase-bromodomain inhibitors for rationally designed polypharmacology. Nat Chem Biol 2014; 10: 305-312.

28 Smith CC, Wang Q, Chin CS, Salerno S, Damon LE, Levis MJ et al. Validation of ITD mutations in FLT3 as a therapeutic target in human acute myeloid leukaemia. Nature 2012; 485: 260-263.

29 Zuber J, Shi J, Wang E, Rappaport AR, Herrmann H, Sison EA et al. RNAi screen identifies Brd4 as a therapeutic target in acute myeloid leukaemia. Nature 2011; 478: $524-528$.

30 Picaud S, Da Costa D, Thanasopoulou A, Filippakopoulos P, Fish PV, Philpott M et al. PFI-1, a highly selective protein interaction inhibitor, targeting BET Bromodomains. Cancer Res 2013; 73: 3336-3346.

31 Dawson MA, Prinjha RK, Dittmann A, Giotopoulos G, Bantscheff M, Chan WI et al. Inhibition of BET recruitment to chromatin as an effective treatment for MLL-fusion leukaemia. Nature 2011; 478: 529-533.

32 Kettenbach AN, Schweppe DK, Faherty BK, Pechenick D, Pletnev AA, Gerber SA. Quantitative phosphoproteomics identifies substrates and functional modules of Aurora and Polo-like kinase activities in mitotic cells. Sci Signal 2011; 4: rs5.

33 Cholewa BD, Desotelle JA, Ahmad N. Small molecule inhibitor of Polo-like kinase 1 (Plk1) causes significant melanoma growth delay and regression in vivo. Cancer Res 2012; 72: 1920.

34 Krause M, Kummer B, Deparade A, Eicheler W, Pfitzmann D, Yaromina A et al. Simultaneous PLK1 inhibition improves local tumour control after fractionated irradiation. Radiother Oncol 2013; 108: 422-428.

35 Lin CC, Su WC, Yen C, Cheng A, Lu Y, Hsu C et al. A phase I dose-escalation study of the polo-like kinase 1 inhibitor volasertib (BI 6727) with two different dosing schedules in patients with advanced solid malignancies. J Clin Oncol 2011; 29: 3046.

36 Stadler WM, Vaughn DJ, Sonpavde G, Vogelzang NJ, Tagawa ST, Petrylak DP et al. An open-label, single-arm, phase 2 trial of the polo-like kinase inhibitor volasertib (BI 6727) in patients with locally advanced or metastatic urothelial cancer. Cancer 2014; 120: 976-982.
37 Pujade-Lauraine E, Weber BE, Ray-Coquard I, Vergote I, Selle F, Del Campo JM et al. Phase II trial of volasertib (BI 6727) versus chemotherapy (CT) in platinumresistant/refractory ovarian cancer (OC). J Clin Oncol 2013; 31: 5504.

38 Ellis PM, Leighl N, Hirsh V, Reaume MN, Blais N, Wierzbicki R et al. A randomised, open-label phase II trial of volasertib as monotherapy and in combination with standard dose pemetrexed compared with pemetrexed monotherapy in secondline non-small cell lung cancer (NSCLC). J Thorac Oncol 2013; 8: 2307.

39 Deleporte A, Dumez H, Awada A, Costermans J, Meeus M, Berghmans $T$ et al. Phase I trial of volasertib (BI 6727), a polo-like kinase 1 (Plk1) inhibitor, in combination with cisplatin or carboplatin in patients with advanced solid tumors. J Clin Oncol 2011; 29: 3031.

40 Dumez H, Gombos A, Schöffski P, Gil T, Vulsteke C, Meeus M-A et al. Phase I trial of the polo-like kinase (Plk) inhibitor volasertib (BI 6727) combined with cisplatin or carboplatin in patients with advanced solid tumors. J Clin Oncol 2012; 30: 3018.

41 Peeters M, Machiels JP, Pilz K, De Smet M, Strelkowa N, Liu D et al. Phase I study of volasertib (BI 6727) combined with afatinib (BIBW 2992) in advanced solid tumors. J Clin Oncol 2013; 31: 2521.

42 De Braud FG, Cascinu S, Spitaleri G, Pilz K, Clementi L, Liu D et al. A phase I dose escalation study of volasertib (BI 6727) combined with nintedanib (BIBF 1120) in advanced solid tumors. J Clin Oncol 2013; 31: 2556.

43 National Comprehensive Cancer Network (NCCN) Clinical Practice Guidelines in Oncology. Acute Myeloid Leukemia. Version 2. Available at: URL http://www.nccn. org (last accessed 10 October 2013).

44 Fey MF, Dreyling M. Acute myeloblastic leukaemias and myelodysplastic syndromes in adult patients: ESMO Clinical Practice Guidelines for diagnosis, treatment and follow-up. Ann Oncol 2010; 21: v158-v161.

45 Döhner H, Estey EH, Amadori S, Appelbaum FR, Büchner T, Burnett AK et al. Diagnosis and management of acute myeloid leukemia in adults: recommendations from an international expert panel, on behalf of the European LeukemiaNet. Blood 2010; 115: 453-474.

46 Ferrara F, Schiffer CA. Acute myeloid leukaemia in adults. Lancet 2013; 381: 484-495.

47 Estey E. AML in older patients: are we making progress? Best Pract Res Clin Haematol 2009; 22: 529-536.

48 Kantarjian H, O'Brien S. Questions regarding frontline therapy of acute myeloid leukemia. Cancer 2010; 116: 4896-4901.

49 Fenaux P, Mufti GJ, Hellstrom-Lindberg E, Santini V, Gattermann N, Germing U et al. Azacitidine prolongs overall survival compared with conventional care regimens in elderly patients with low bone marrow blast count acute myeloid leukemia. J Clin Oncol 2010; 28: 562-569.

50 Burnett AK, Milligan D, Prentice AG, Goldstone AH, McMullin MF, Hills RK et al. A comparison of low-dose cytarabine and hydroxyurea with or without all-trans retinoic acid for acute myeloid leukemia and high-risk myelodysplastic syndrome in patients not considered fit for intensive treatment. Cancer 2007; 109: 1114-1124.

51 Kantarjian HM, Thomas XG, Dmoszynska A, Wierzbowska A, Mazur G, Mayer J et al. Multicenter, randomized, open-label, phase III trial of decitabine versus patient choice, with physician advice, of either supportive care or low-dose cytarabine for the treatment of older patients with newly diagnosed acute myeloid leukemia. J Clin Oncol 2012; 30: 2670-2677.

52 Yanada M, Naoe T. Acute myeloid leukemia in older adults. Int J Hematol 2012; 96 186-193.

53 Ikezoe T, Yang J, Nishioka C, Takezaki Y, Tasaka T, Togitani $\mathrm{K}$ et al. A novel treatment strategy targeting polo-like kinase 1 in hematological malignancies. Leukemia 2009; 23: 1564-1576.

54 Bug G, Schlenk RF, Müller-Tidow C, Lübbert M, Krämer A, Fleischer F et al. Phase I/ II study of BI 6727 (volasertib), an intravenous polo-like kinase-1 (Plk1) inhibitor, in patients with acute myeloid leukemia (AML): results of the dose finding for $\mathrm{BI}$ 6727 in combination with low-dose cytarabine. Blood 2010; 116: abstract 3316.

55 Bug G, Müller-Tidow C, Schlenk RF, Krämer A, Lübbert M, Krug U et al. Phase I/II study of volasertib (BI 6727), an intravenous polo-like kinase (PIk) inhibitor, in patients with acute myeloid leukemia $(A M L)$ : updated results of the dose finding phase I part for volasertib in combination with low-dose cytarabine (LD-Ara-C) and as monotherapy in relapsed/refractory AML. Blood 2011; 118: abstract 1549.

56 Döhner H, Bug G, Müller-Tidow C, Krämer A, Lübbert M, Krug U et al. Phase I/II study of volasertib, an intravenous Polo-like kinase inhibitor (Plk), in patients with relapsed/refractory acute myeloid leukemia (AML): updated phase I results for volasertib monotherapy. Haematologica 2014; 99: abstract S649.

57 Döhner H, Lübbert M, Fiedler W, Fouillard L, Haaland A, Brandwein JM et al. Randomized, phase 2 trial comparing low-dose cytarabine with or without volasertib in AML patients not suitable for intensive induction therapy. Blood 2014; e-pub ahead of print 8 July 2014; doi: http://dx.doi.org/10.1182/blood-2014 03-560557. 
58 Chen YJ, Lin YP, Chow LP, Lee TC. Proteomic identification of Hsp70 as a new Plk1 substrate in arsenic trioxide-induced mitotically arrested cells. Proteomics 2011; 11: 4331-4345.

59 Renner AG, Creancier L, Dos Santos C, Fialin C, Recher C, Bailly C et al. A functional link between polo-like kinase 1 and the mammalian target-of-rapamycin pathway? Cell Cycle 2010; 9: 1690-1696.

60 Younes A, Samad N. Utility of mTOR inhibition in hematologic malignancies. Oncologist 2011; 16: 730-741.

61 Park S, Chapuis N, Tamburini J, Bardet V, Cornillet-Lefebvre P, Willems L et al. Role of the PI3K/AKT and mTOR signaling pathways in acute myeloid leukemia. Haematologica 2010; 95: 819-828.

62 Récher C, Dos Santos C, Demur C, Payrastre B. mTOR: a new therapeutic target in acute myeloid leukemia. Cell Cycle 2005; 4: 1540-1549.

63 Oppermann FS, Grundner-Culemann K, Kumar C, Gruss OJ, Jallepalli PV, Daub H. Combination of chemical genetics and phosphoproteomics for kinase signaling analysis enables confident identification of cellular downstream targets. Mol Cell Proteomics 2012; 11: 0111.012351.

64 Schaab C, Oppermann F, Pfeifer H, Klammer M, Tebbe A, Oellerich T et al. Global phosphoproteome analysis of AML bone marrow reveals predictive markers for the treatment with AC220. Blood 2012; 120: 786.

65 Hjelle SM, Forthun RB, Haaland I, Reikvam H, Sjoholt G, Bruserud O et al. Clinical proteomics of myeloid leukemia. Genome Med 2010; 2: 41.

66 Skavland J, Jorgensen KM, Hadziavdic K, Hovland R, Jonassen I, Bruserud O et al. Specific cellular signal-transduction responses to in vivo combination therapy with ATRA, valproic acid and theophylline in acute myeloid leukemia. Blood Cancer J 2011; 1: e4.

67 Anensen N, Hjelle SM, Van BW, Haaland I, Silden E, Bourdon JC et al. Correlation analysis of p53 protein isoforms with NPM1/FLT3 mutations and therapy response in acute myeloid leukemia. Oncogene 2012; 31: 1533-1545.

68 Lee M, Daniels MJ, Venkitaraman AR. Phosphorylation of BRCA2 by the Polo-like kinase Plk1 is regulated by DNA damage and mitotic progression. Oncogene 2004; 23: $865-872$.
69 Degenhardt Y, Greshock J, Laquerre S, Gilmartin AG, Jing J, Richter M et al. Sensitivity of cancer cells to Plk1 inhibitor GSK461364A is associated with loss of p53 function and chromosome instability. Mol Cancer Ther 2010; 9: 2079-2089.

70 Sanhaji M, Kreis NN, Zimmer B, Berg T, Louwen F, Yuan J. p53 is not directly relevant to the response of Polo-like kinase 1 inhibitors. Cell Cycle 2012; 11: 543-553.

71 Dawson MA, Gudgin EJ, Horton SJ, Giotopoulos G, Meduri E, Robson S et al. Recurrent mutations, including NPM1c, activate a BRD4-dependent core transcriptional program in acute myeloid leukemia. Leukemia 2014; 28: 311-320.

72 Stewart HJ, Horne GA, Bastow S, Chevassut TJ. BRD4 associates with p53 in DNMT3A-mutated leukemia cells and is implicated in apoptosis by the bromodomain inhibitor JQ1. Cancer Med 2013; 2: 826-835.

73 Tie F, Banerjee R, Conrad PA, Scacheri PC, Harte PJ. Histone demethylase UTX and chromatin remodeler BRM bind directly to $\mathrm{CBP}$ and modulate acetylation of histone H3 lysine 27. Mol Cell Biol 2012; 32: 2323-2334.

74 Forthun RB, Sengupta T, Skjeldam HK, Lindvall JM, McCormack E, Gjertsen BT et al. Cross-species functional genomic analysis identifies resistance genes of the histone deacetylase inhibitor valproic acid. PLoS One 2012; 7: e48992.

75 Jain RK. Vascular and interstitial barriers to delivery of therapeutic agents in tumors. Cancer Metastasis Rev 1990; 9: 253-266.

(c) (i) This work is licensed under a Creative Commons Attribution 4.0 International License. The images or other third party material in this article are included in the article's Creative Commons license, unless indicated otherwise in the credit line; if the material is not included under the Creative Commons license, users will need to obtain permission from the license holder to reproduce the material. To view a copy of this license, visit http://creativecommons.org/licenses/ by/4.0/ 